\title{
Studies with Cathode Drift Chambers for the GlueX Experiment at Jefferson Lab th
}

\author{
L. Pentchev ${ }^{\mathrm{a}, *}$, F. Barbosa ${ }^{\mathrm{a}}$, V. Berdnikov ${ }^{\mathrm{b}}$, D. Butler ${ }^{\mathrm{a}}$, S. Furletov ${ }^{\mathrm{a}}, \mathrm{L}$. \\ Robison $^{\mathrm{c}}$, B. Zihlmann ${ }^{\mathrm{a}}$ \\ ${ }^{a}$ Thomas Jefferson National Accelerator Facility, Newport News, Virginia 23606, USA \\ ${ }^{b}$ National Research Nuclear University MEPhi, Moscow, Russia \\ ${ }^{c}$ Northwestern University, Evanston, IL 60208, USA
}

\begin{abstract}
A drift chamber system consisting of $241 \mathrm{~m}$-diameter chambers with both cathode and wire readout (total of 12,672 channels) is operational in Hall D at Jefferson Lab (Virginia). Two cathode strip planes and one wire plane in each chamber register the same avalanche allowing the study of avalanche development, charge induction process, and strip resolution. We demonstrate a method for reconstructing the two-dimensional distribution of the avalanche "center-ofgravity" position around the wire from an ${ }^{55} \mathrm{Fe}$ source with resolutions down to $30 \mu \mathrm{m}$. We estimate the azimuthal extent of the avalanche around the wire as a function of the total charge for an $\mathrm{Ar} / \mathrm{CO}_{2}$ gas mixture. By means of cluster counting using a modified $3 \mathrm{~cm}$-gap chamber, we observe significant space charge effects within the same track, resulting in an extent of the avalanche along the wire.
\end{abstract}

Keywords: tracking, drift chamber, avalanche, cluster counting

\footnotetext{
${ }^{\text {*T }}$ Authored by Jefferson Science Associates, LLC under U.S. DOE Contract No. DE-AC0506OR23177. The U.S. Government retains a non-exclusive, paid-up, irrevocable, world-wide license to publish or reproduce this manuscript for U.S. Government purposes.

* Corresponding author

Email address: pentchev@jlab.org (L. Pentchev)
}

Preprint submitted to Nuclear Instruments and Methods

April 18, 2016

(C) 2016. This manuscript version is made available under the Elsevier user license http://www.elsevier.com/open-access/userlicense/1.0/ 


\section{Introduction}

The GlueX experiment [1] uses tagged polarized photons from the recently upgraded $12 \mathrm{GeV}$ Continuous Electron Beam Accelerator Facility (CEBAF) to search for exotic hybrid mesons (quantum numbers not allowed as quark-antiquark 5 configuration), as predicted by lattice QCD. The identification of such mesons requires full reconstruction of all charged particles and photons in the event with high position and momentum resolution. The GlueX detector has a hermetic coverage (to optimize the partial wave amplitude analysis) for charged and neutral particles using calorimetry and tracking within a $2 \mathrm{~T}$-solenoid. The tracking in the forward region requires resolutions of $150 \mu \mathrm{m}$ and a good multitrack separation. This is achieved with planar drift chambers with additional cathode strips on both sides of the wire plane and multihit pipelined readout. Some important advantages of such redundancy in the hit registration is the possibility to work with high track densities, to operate at low thresholds very close to the non-correlated noise level, and to "self-calibrate" the detector 1 At the same time, such a chamber configuration allows to study the avalanche development and corresponding signal formation, as presented here. Chambers with special multi-electrode design have been used for similar studies (though for different gases) before, as presented in these classical works, 2], 3], 4]. In our studies we have used full-scale detectors operated in the experiment, taking advantage of modern pipelined electronics, with the main purpose to better understand and improve the detector resolution.

\section{The detector}

Twenty-four drift chambers of 1m-diameter, grouped into four packages, are located within the bore of the spectrometer magnet. Each drift chamber consists of two cathode planes divided into strips at $5 \mathrm{~mm}$ on both sides of the wire plane

\footnotetext{
${ }^{1}$ Full description of the detector and its performance within the GlueX experiment will follow this publication.
} 
(Fig 1). The wire plane has sense $(20 \mu \mathrm{m})$ and field wires $(80 \mu \mathrm{m}) 5 \mathrm{~mm}$ apart. The strips are $2-\mu \mathrm{m}$ thick copper with a pitch of $5 \mathrm{~mm}$. The strips on the two cathodes are oriented at different angles w.r.t. wires: $75^{0}$ and $105^{\circ}$. The 30 frames are light, made mostly of Rohacell with a G10 skin, to allow detection of low energy photons by the outside electro-magnetic calorimeters. Charge pre-

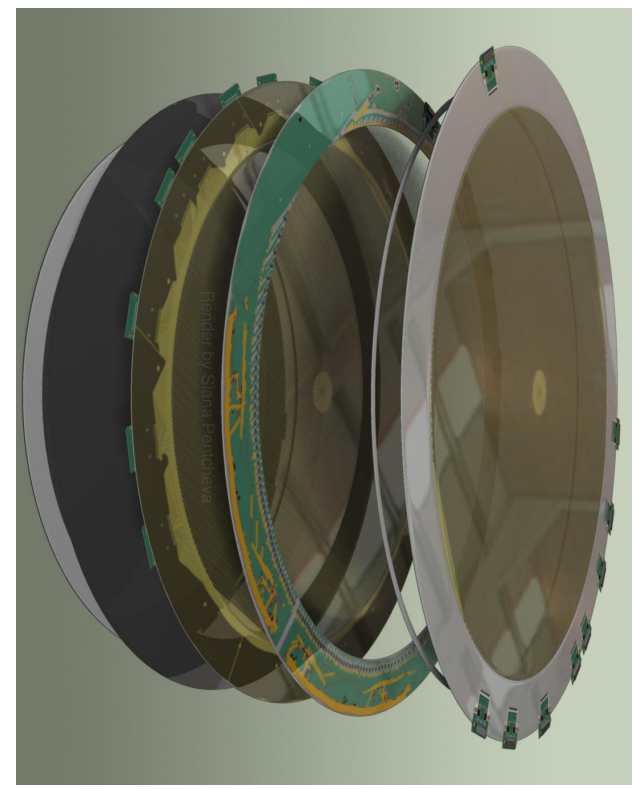

Figure 1: Artistic view of one chamber (out of 24). From right to left: upstream cathode, spacer ring, wire plane, downstream cathode, aluminized Mylar separating the chambers.

amplifiers with $10 \mathrm{~ns}$ peaking time are used to read the signals from 2,304 wires and 10,368 strips. The data are pipelined by means of TDCs for the wires and $125 \mathrm{MHz}$-flashADCs for the strips. The cathode drift chamber system has been operational for more than a year within the GlueX experiment that is already producing physics results.

\section{Hit reconstruction and strip resolution}

The avalanche around the wire is seen in three projections - two cathode planes and the wire plane. The angles of the strips in the two cathodes are 40 optimized to reconstruct the avalanche/hit position along the wire. The drift 
times from the wire signals are used to reconstruct the hit position in direction perpendicular to the wire. Thus, each chamber gives a hit position in 3D space. This allows us to reconstruct tracks at high density close to the beam line.

The two cathodes can also reconstruct the wire positions (i.e. avalanche positions in the direction perpendicular to the wire), although with a poorer resolution due to the unfavored angle of the strips w.r.t. the wires. This is a powerful tool to correct the geometry of the strips by requiring the cathodereconstructed wires to be straight and at the right positions over the whole area of the chamber. At the same time, the widths of the wire position distributions are used to estimate the strip resolution, after correction for the strip angle which is a factor of $\sqrt{2} \cos \left(75^{\circ}\right)=0.366$. Fig 2 shows the measured widths and corresponding strip resolutions (i.e. in the direction perpendicular to the strips) as a function of the total charge obtained with beam from reconstructed particle tracks. Generally, the resolution improves with increasing total charge.

Cathode resolutions from wire position reconstruction

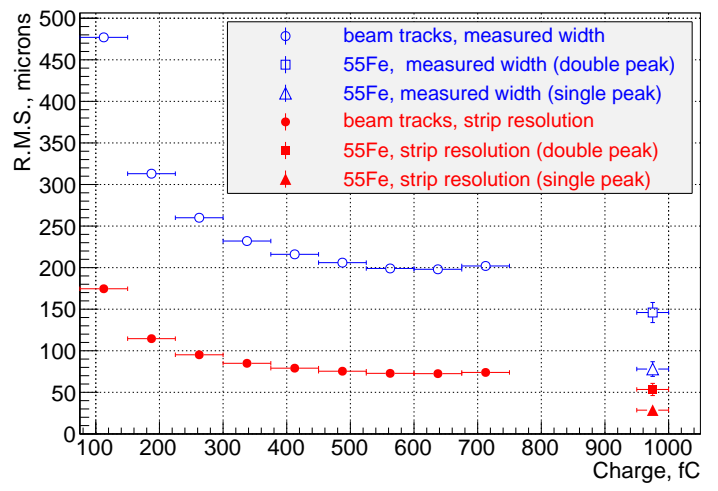

Figure 2: Measured widths of the wire positions and estimated (see text) strip resolutions as a function of the total charge from wire position reconstruction using tracks and an ${ }^{55} \mathrm{Fe}$ source.

\section{Avalanche position reconstruction}

We take advantage of the high charge and fixed signal shape when using an ${ }^{55} \mathrm{Fe}$ source to further study the strip resolution. The studies are done with an 
$\mathrm{Ar} / \mathrm{CO}_{2} 40 / 60 \%$ gas mixture. In this section we use the strips from the two cathodes to reconstruct the avalanche position in the direction perpendicular to the wire ( $x$-coordinate). Furthermore, by taking the ratio of the total charges on the two cathodes we obtain with very high precision the avalanche position in the direction perpendicular to the cathodes ( $z$-coordinate). As a result we reconstruct the $2 \mathrm{D}$ distribution of the avalanche position around the wire - Fig 3 The electric field configuration is such that the area with field lines that end on the field wires and start from the left/right side of the sense wires is much smaller than the area with field lines ending on the cathodes. This explains the smaller number of events at 3 and 9 o'clock in Fig 3 . In addition, due to the $\mathrm{keV}$-photon attenuation in the gas, the densities are higher on the side closer to the ${ }^{55} \mathrm{Fe}$ source. Plotted is the apparent avalanche position as the center-of-gravity seen

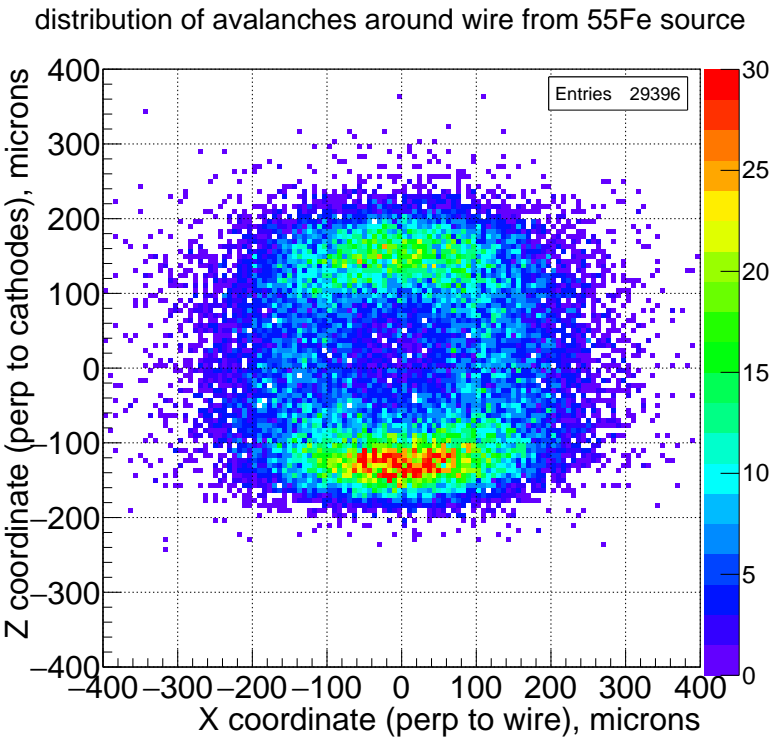

Figure 3: Avalanche position around the wire reconstructed from strip crossing ( $x$-coordinate) and cathode charge ratio ( $z$-coordinate). Uncollimated ${ }^{55} \mathrm{Fe}$ source placed at bottom side, $\mathrm{Ar} / \mathrm{CO}_{2}$ 40/60\%.

70 by the strips. The actual avalanche position is different due to the distortion of 
the charge induction as a result of the proximity of the avalanche to the wire; the corresponding corrections are not applied for this plot. Slices in $x$ and $z$ of the above distribution are shown on Figs 4 and 5 . The two-peak structure in the $x$-distribution is visible only because we have measured the $z$-coordinate simultaneously and cut a slice at $z=0$. To compare with the results from the previous section for measurements with beam, we plot in Fig 2 the R.M.S. of the two peak structure and separately the single-peak resolution. Similarly to the beam measurements, we estimate the corresponding strip resolutions that are shown on the same plot.

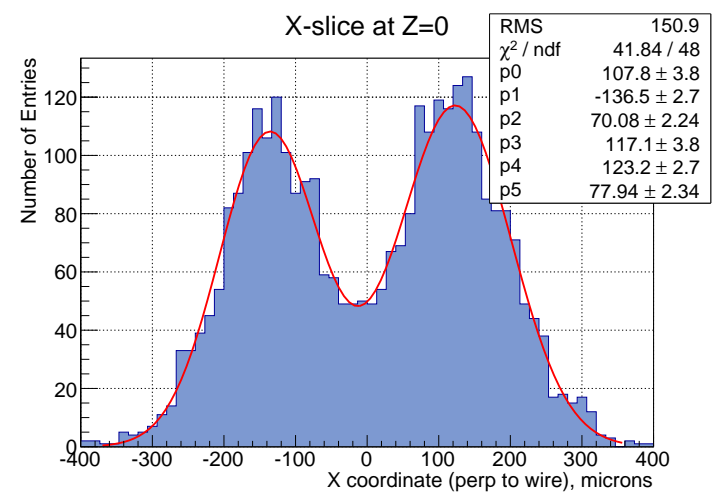

Figure 4: Slice of the $2 \mathrm{D}$ distribution in Fig 3 at $\mathrm{Z}=0$ fitted with two Gaussians. $p 2$ and $p 5$ parameters are the corresponding avalanche position resolutions.

Needless to say, such measurements require very good calibration of the strip signals. Since the strips are at an angle w.r.t. the wire, for a fixed wire, different strips share the charge for different avalanche positions along the wire. As an example, the top panel in Fig 6 shows the cathode charge ratio as calculated from the raw strip signals for one particular wire fired, as a function of the avalanche position along the wire. The fine structure in the ratio is a result of a systematic effect in the charge integration that depends on the position of the avalanche w.r.t. the middle of the corresponding strip. After correcting for this, we require the ratio to be constant along the wire with the two peaks positioned symmetrically w.r.t. unity, which effectively calibrates the charges 


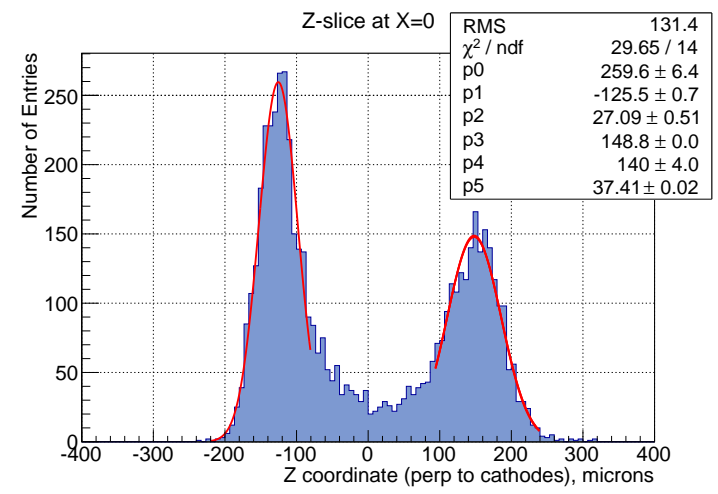

Figure 5: Slice of the 2D distribution in Fig 3 at $\mathrm{X}=0$ fitted with two Gaussians in limited regions. $p 2$ and $p 5$ parameters are the corresponding avalanche position resolutions.

for the different strips. The corrected ratio is shown at the bottom plot in Fig6. Similar corrections are done for the $x$-coordinate (wire position) which is required to be constant along the wire. In other words, the procedure described here is "self-calibrating", allowing us to achieve such high resolutions.

The two peaks in the cathode charge ratio for $x=0$ (i.e. at 12 and 6 o'clock in Fig 3 are plotted in Fig 7 a as a function of the total charge for different HV settings of the sense and field wires. Depending on which side the avalanche is w.r.t. the wire, there is an asymmetry in the charge ratio, which is also predicted by Garfield [5] simulations as shown in the same figure. This asymmetry is due to an asymmetry in the weighting field around the wire when the potential of one of the cathodes is set to unity. However, the observed dependence of the charge asymmetry on the total charge, as also seen in [3], is not predicted by Garfield. Such dependence can be explained by the azimuthal extent of the avalanche around the wire. Since this is mostly a photon-mediated process (4]) it is not taken into account in Garfield [5]. To simulate it, we distribute electrons evenly around the wire within a certain azimuthal range, and propagate their avalanches with Garfield. We vary the range until we match the measured cathode charge ratio with the the simulated ratio. Example of such simulation for an azimuthal range of $270^{\circ}$ is shown in Fig 8 . Thus, we estimate 

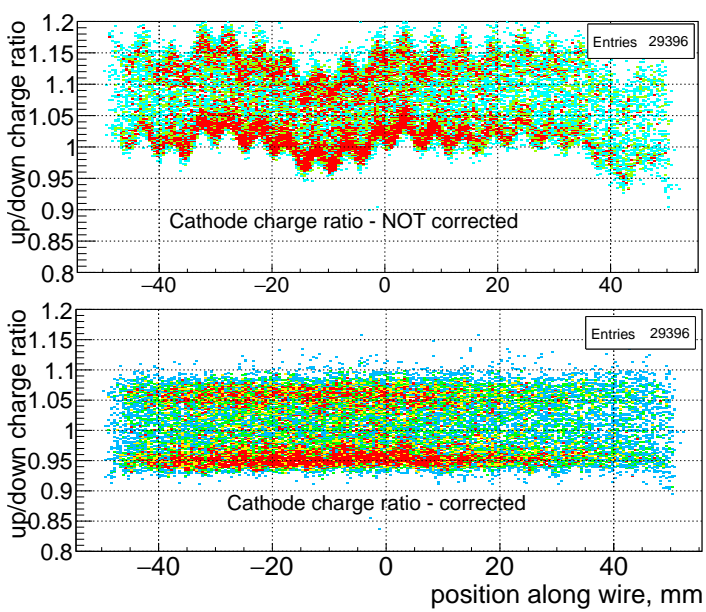

Figure 6: Ratio of the total charge on the upper and lower cathodes as a function of the position along one wire without corrections (top) and with corrections (bottom) as explained in the text; using ${ }^{55} \mathrm{Fe}$ source.

the dependence of the azimuthal range of the avalanche origin on the charge ratio and in turn, from Fig $7 \mathrm{a}$, the dependence on the total charge (Fig $7 \mathrm{~b}$ ). The direct measurements of the azimuthal extent [4, using a special segmented cathode tube, are in qualitative agreement with our results, though for different gas mixtures.

Similar studies can be done for the avalanches at $z=0$ (3 and 9 o'clock). The poorer resolution in this direction (compare Figs 4 and 5), especially for low gains, prevents us from obtaining conclusive results. In addition, in contrast to the total charge simulation, the calculations of the weighting field for the individual strips and consequently of the induced signals, are much more complicated [6].

\section{Cluster counting studies}

One chamber has been modified to a $3 \mathrm{~cm}$ drift gap and operated at a gas gain of about $2 \cdot 10^{5}$. High single electron efficiency and long drift times allowed counting of the number of clusters for each track using flash-ADCs [7. The 

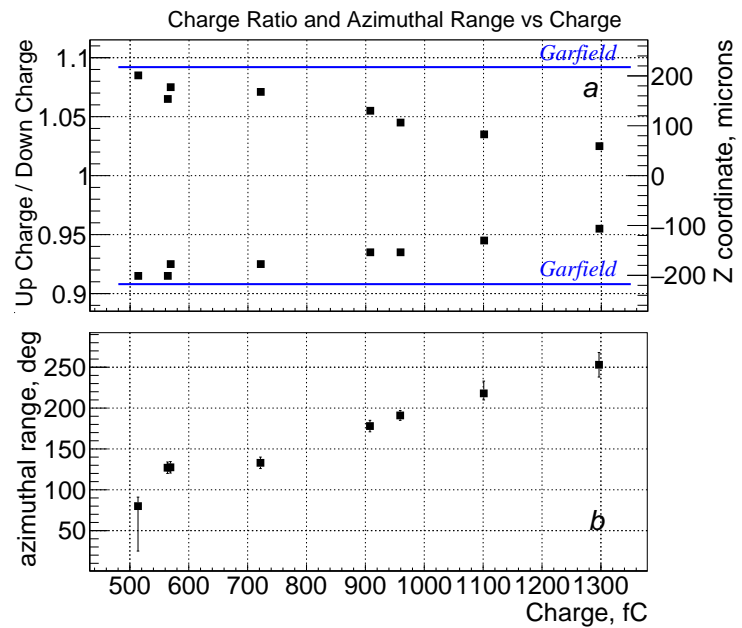

Figure 7: (a) Cathode charge ratio (data points) for avalanches at 12 and 6 o'clock $(x=0)$ as function of the total charge. The two lines represent Garfield predictions. The "center-ofmass" z-position is shown on the right vertical scale. (b) Azimuthal range of the avalanche origin around the wire as function of the total charge; $\mathrm{Ar} / \mathrm{CO}_{2} 40 / 60 \%$.

number of clusters turned out to be a strong function of the track angle w.r.t. the wire (Fig 9). For tracks perpendicular to the wire the electrons travel almost along the same path and encounter higher space charge near the wire from the previous electrons. This can explain the lower number of clusters for such tracks. A rough estimate of the average dimension of the space charge along the wire, of at least $300 \mu \mathrm{m}$, can be inferred from the plot. The fluctuations in such a process may affect the strip resolution along the wire.

\section{Conclusions}

The redundancy in avalanche reconstruction allows operation at low threshold and precise calibration, reaching resolutions of better than $150 \mu \mathrm{m}$ with tracks. The multi-electrode chamber configuration and the use of an ${ }^{55} \mathrm{Fe}$ source gives us a tool to study the intrinsic resolution of the detector due to the finite extent of the avalanche. We reconstruct the position of the avalanche w.r.t. the wire in two dimensions with resolutions down to $30 \mu \mathrm{m}$. The ratio of the 


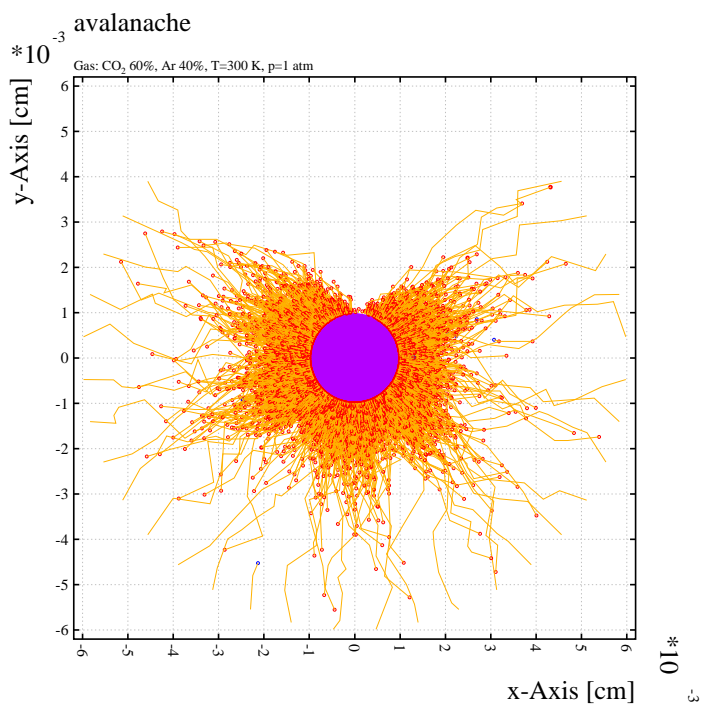

Figure 8: Garfield simulations of the avalanche development with primary electrons evenly distributed in $270^{\circ}$-wide azimuthal range.

charges on the two cathodes depends on the total charge. This can be explained by photon-mediated azimuthal extent of the avalanche, which has been quantitatively estimated for an $\mathrm{Ar} / \mathrm{CO}_{2}$ gas mixture. Cluster counting studies with $3 \mathrm{~cm}$-gap modified chamber show sharp decline in the cluster number for tracks perpendicular to the wire, demonstrating the effect of the space charge within the same track.

We thank E. Chudakov for his support and discussions and B. Wojtsekhowski for his important remarks. This material is based upon work supported by the U.S. Department of Energy, Office of Science, Office of Nuclear Physics under contract DE-AC05-06OR23177.

\section{References}

ㅁ1] JLab Experiment E12-06-102 https://www.jlab.org/exp_prog/proposals/06/PR1206-102.pdf, 2006. 


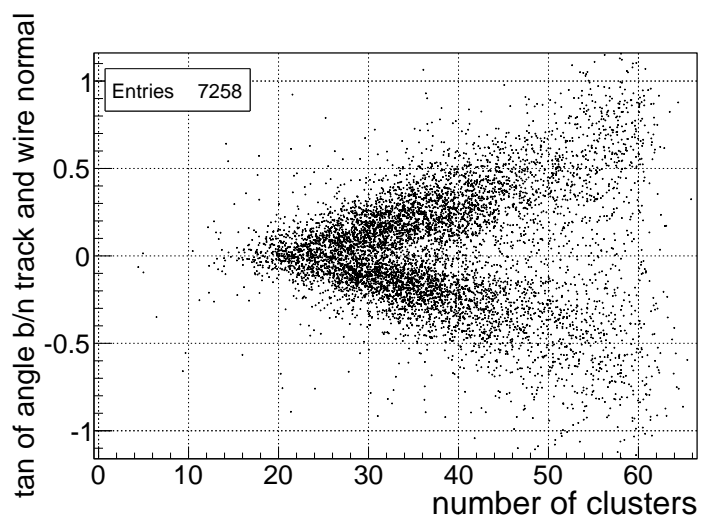

Figure 9: Tangent of the angle between the track and the wire normal plane vs reconstructed number of clusters; $\mathrm{Ar} / \mathrm{CO}_{2} 60 / 40 \%$.

[2] G. Charpak, G. Petersen, A. Policarpo and F. Sauli, Nuclear Instruments and Methods in Physics Research, 148 (1978) 471.

[3] J. Fisher, H. Okuno and A.H. Walenta, Nuclear Instruments and Methods in Physics Research, 151 (1978) 451.

[4] H. Okuno, J. Fisher, V. Radeka, A.H. Walenta, IEEE Trans. Nucl. Sci. NS-26 (1979) 160.

[5] R. Veenhof, GARFIELD simulation tool, http://cern.ch/garfield

[6] J.S. Gordon and E. Mathieson, Nuclear Instruments and Methods in Physics Research, 227 (1984) 267; E. Mathieson, Nuclear Instruments and Methods in Physics Research, A270 (1988) 602.

[7] V.V. Berdnikov, S.V. Somov and L. Pentchev, Instrum.Exp.Tech. 58 no.4 (2015) 473. 